\title{
Photoquantum Hall Effect and Light-Induced Charge Transfer at the Interface of Graphene/InSe Heterostructures
}

\author{
Mahabub A. Bhuiyan, Zakhar R. Kudrynskyi, Debarati Mazumder, Jake D. G. Greener, \\ Oleg Makarovsky, Christopher J. Mellor, Evgeny E. Vdovin, Benjamin A. Piot, Inna I. Lobanova, \\ Zakhar D. Kovalyuk, Marina Nazarova, Artem Mishchenko, Kostya S. Novoselov, Yang Cao, \\ Laurence Eaves, Go Yusa, and Amalia Patanè*
}

The transfer of electronic charge across the interface of two van der Waals crystals can underpin the operation of a new class of functional devices. Among van der Waals semiconductors, an exciting and rapidly growing development involves the "post-transition" metal chalcogenide InSe. Here, field effect phototransistors are reported where single layer graphene is capped with n-type InSe. These device structures combine the photosensitivity of InSe with the unique electrical properties of graphene. It is shown that the light-induced transfer of charge between InSe and graphene offers an effective method to increase or decrease the carrier density in graphene, causing a change in its resistance that is gate-controllable and only weakly dependent on temperature. The charge transfer at the InSe/graphene interface is probed by Hall effect and photoconductivity measurmentes and it is demonstrated that light can induce a sign reversal of the quantum Hall voltage and photovoltaic effects in the graphene layer. These findings demonstrate the potential of light-induced charge transfer in gate-tunable InSe/graphene phototransistors for optoelectronics and quantum metrology.

\section{Introduction}

Atomically thin layers of van der Waals (vdW) crystals and their heterostructures offer unique opportunities to explore and manipulate the transfer of electronic charge at the interface of different materials and to create highperformance multi-functional devices for integration with photonic and electronic technologies. ${ }^{[1]}$ VdW crystals have strong covalent bonding within the 2D atomic planes and weak vdW attraction between the layers. These properties enable the fabrication of stable films down to monolayer thickness and the realization of a wide range of multilayered structures, overcoming limitations of traditional semiconductor heterojunctions, such as incompatibility of the active layer and substrate due to lattice mismatch,
M. A. Bhuiyan, Dr. Z. R. Kudrynskyi, D. Mazumder, J. D. G. Greener,

Dr. O. Makarovsky, Dr. C. J. Mellor, Dr. E. E. Vdovin,

Prof. L. Eaves, Prof. A. Patanè

School of Physics and Astronomy

The University of Nottingham

Nottingham NG7 2RD, UK

E-mail: amalia.patane@nottingham.ac.uk

Dr. E. E. Vdovin

Institute of Microelectronics Technology and High Purity Materials

RAS

Chernogolovka 142432, Russia

Dr. B. A. Piot, Dr. I. I. Lobanova

Laboratoire National des Champs Magnétiques Intenses

CNRS-UGA-UPS-INSA-EMFL

25 rue des Martyrs 38042, Grenoble, France

Prof. Z. D. Kovalyuk

Institute for Problems of Materials Science

The National Academy of Sciences of Ukraine

Chernivtsi Branch, Chernivtsi 58001, Ukraine

The ORCID identification number(s) for the author(s) of this article can be found under https://doi.org/10.1002/adfm.201805491.
M. Nazarova, Dr. A. Mishchenko, Prof. K. S. Novoselov

School of Physics and Astronomy

University of Manchester

Oxford Road, Manchester M13 9PL, UK

M. Nazarova, Dr. Y. Cao

National Graphene Institute

University of Manchester

Oxford Road, Manchester M13 9PL, UK

Dr. Y. Cao

State Key Laboratory of Physical Chemistry of Solid Surface

College of Chemistry and Chemical Engineering

Collaborative Innovation Center of Chemistry for Energy Materials

Xiamen University

Xiamen 361005, China

Dr. G. Yusa

Department of Physics

Tohoku University

Sendai 980-8578, Japan

DOI: 10.1002/adfm.201805491 
defective interfaces, etc. ${ }^{[2]}$ Ongoing research is exploring a wide range of $2 \mathrm{D}$ materials such as graphene, the wide-gap insulator hexagonal boron nitride $(\mathrm{hBN}){ }^{[1,2]}$ the narrow band gap semiconductor black phosphorus (bP), ${ }^{[3,4]}$ and transition metal dichalcogenides (TMDCs, e.g., $\mathrm{MoS}_{2}, \mathrm{WS}_{2}$, etc.). ${ }^{[5,6]}$ Among these 2D materials, the "post-transition" metal monochalcogenides, such as InSe, are receiving increasing attention. ${ }^{[7-19]}$ The $\gamma$-polytype phase of InSe has a band gap that increases markedly with decreasing layer thickness down to a single layer and an interband optical spectrum ranging from the violet to the infrared. ${ }^{[7-9]}$ It has a Brillouin zone-centered conduction band with a relatively small in-plane electron effective mass $\left(m_{\mathrm{c}} \sim 0.14 m_{\mathrm{e}}\right)^{[7]}$ that has been exploited in high mobility $\left(\mu=0.2 \mathrm{~m}^{2} \mathrm{~V}^{-1} \mathrm{~s}^{-1}\right.$ at $300 \mathrm{~K}$ and $2 \mathrm{~m}^{2} \mathrm{~V}^{-1} \mathrm{~s}^{-1}$ at $2 \mathrm{~K}) 2 \mathrm{D}$ field effect transistors $(\mathrm{FETs})^{[9,10]}$ and devices with quantum point contacts or electrostatically defined quantum dots. ${ }^{[11,18]}$ In particular, InSe can act as an electrically and optically active layer to "modulation-dope"[13] and photosensitize $^{[14]}$ an adjacent graphene layer, and to induce a "giant" quantum Hall (QH) plateau in its magnetoresistance. ${ }^{[13]}$ These phenomena involve a transfer of electronic charge at the InSe/graphene interface and offer opportunities to explore a diverse range of new electrical and optical devices.

Here we report on the effects of light on the transport properties of field effect phototransistors in which a layer of graphene is partially capped with n-type InSe. We use a combination of optical and magneto-transport studies to demonstrate that the absorption of light by the InSe induces: i) either an increase or decrease of resistance in the graphene layer that is gate-tuneable, but only weakly affected by temperature in the range $T=2-300 \mathrm{~K}$; ii) a reversal in the sign of the Hall voltage in the $\mathrm{QH}$ regime that can be controlled by an appropriate combination of gate voltages and optical illumination over a range of temperatures of up to $\approx 200 \mathrm{~K}$; iii) photovoltaic effects due to a built-in electric field at the graphene/InSe interface. These effects arise from the sensitivity of the electrical conductivity of the graphene layer to electronic charges that are photogenerated in the InSe and transferred across the interface onto the graphene. This light-induced charge transfer offers an effective method to increase or decrease selectively the carrier density in the InSe-capped graphene. An optical induced transition from a hole- to an electron-carrier current is also achieved over an extended range of gate voltages, with potential for quantum metrology and high-photosensitive sensors and switches.

\section{Results and Discussion}

\subsection{Gate-Tunable Graphene Transistors Photosensitized with InSe}

Figure 1a shows an optical image of one of our InSe/graphene heterostructure devices. The InSe layer forms a cap over one half of the graphene Hall bar. The two sections of the device comprising uncapped and InSe-capped graphene are in a "series" resistance configuration with the same electrical current, I, flowing through both of them (Figure 1b). Magnetotransport experiments were performed in two modes: i) in darkness and ii) with the device uniformly illuminated by an unfocused He-Ne laser beam with a photon energy, $h v=1.96 \mathrm{eV}$, in excess of the bandgap energy of bulk InSe, $E_{\mathrm{g}}=1.26 \mathrm{eV}$ at $300 \mathrm{~K}$ (see the Experimental Section). The $V_{\mathrm{g}}$-dependence of the low $(T=2 \mathrm{~K})$ and room temperature $(T=300 \mathrm{~K})$ resistance, $R_{x x}$, of the uncapped and InSe-capped graphene was measured at zero applied magnetic field in darkness, see black curves in Figure 1c. For uncapped graphene, $R_{x x}$ reaches a maximum at a positive gate voltage, $V_{\mathrm{g}}=+21 \mathrm{~V}$ at $T=2 \mathrm{~K}$, from which we deduce that the graphene is $p$-doped at $V_{\mathrm{g}}=0 \mathrm{~V}$ with $p=1.6 \times 10^{12} \mathrm{~cm}^{-2}$ (Supporting Information S1). For InSe-capped graphene, the maximum of $R_{x x}\left(V_{\mathrm{g}}\right)$ occurs at a lower gate voltage, $V_{\mathrm{g}}=+7 \mathrm{~V}$, shifted by $\Delta V_{\mathrm{g}} \approx-14 \mathrm{~V}$ relative to the peak for uncapped graphene, corresponding to a reduced hole density. The $R_{x x}\left(V_{\mathrm{g}}\right)$ curve for the graphene-only section of the Hall bar does not change when its surface is illuminated with laser light. In contrast, for InSe-capped graphene, the maximum of $R_{x x}\left(V_{\mathrm{g}}\right)$ shifts toward lower gate voltages at both $T=2$ and $300 \mathrm{~K}$ (red curves in Figure 1c). This shift, $\Delta V_{\mathrm{g}}$, is proportional to the light intensity at low levels, but saturates at
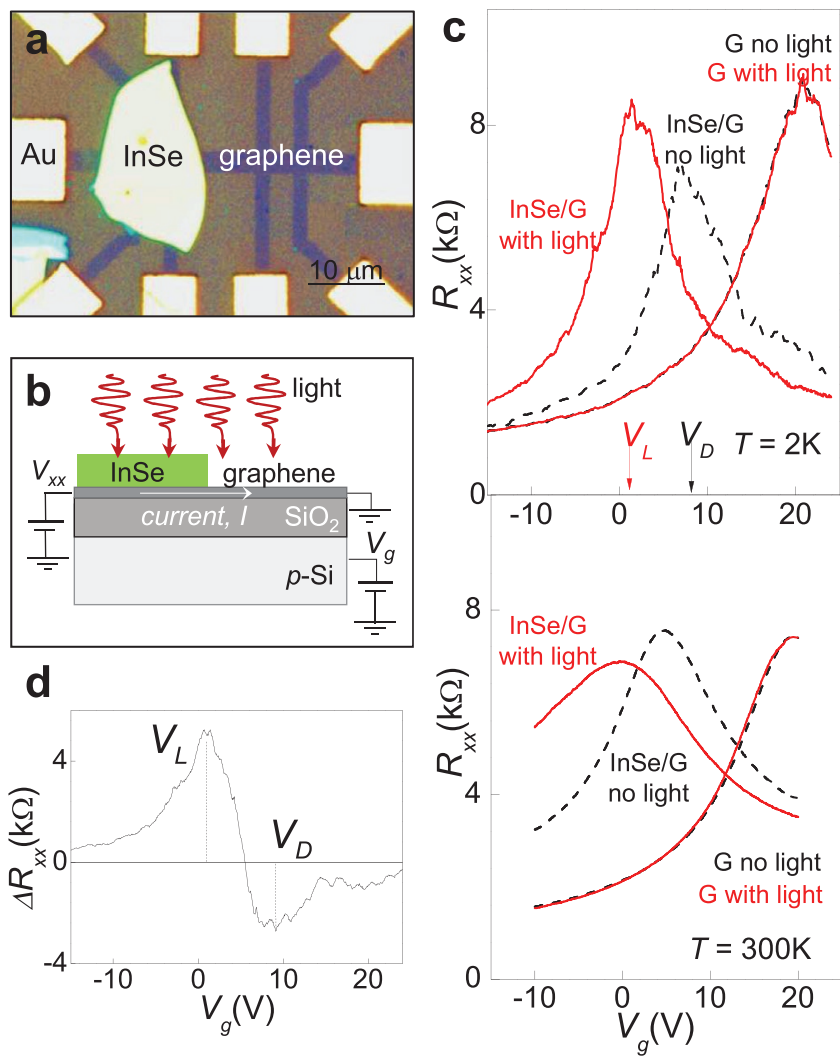

Figure 1. a) Optical image and b) sketch of a Hall bar containing uncapped and InSe-capped graphene. The thickness of the InSe layer is $50 \mathrm{~nm}$, as measured by atomic force microscopy. Devices with different layer thickness down to $\approx 10 \mathrm{~nm}$ show a similar behavior. c) Resistance $R_{x x}$ versus gate bias $V_{\mathrm{g}}(B=0 \mathrm{~T}$ and $I=0.5 \mu \mathrm{A})$ without (black curves) and with illumination (red curves; $\lambda=633 \mathrm{~nm}$ and $P=9 \mathrm{~mW} \mathrm{~mm}$ ) for uncapped graphene $(G)$ and InSe-capped graphene $(\operatorname{InSe} / \mathrm{G})$. Top: $T=2 \mathrm{~K}$; bottom: $T=300 \mathrm{~K}$. $V_{\mathrm{L}}$ and $V_{\mathrm{D}}$ denote the peak position of the $R_{x x}\left(V_{\mathrm{g}}\right)$ curves under illumination and in darkness, respectively. d) Dependence on the gate voltage $V_{\mathrm{g}}$ of the change in the resistance, $\Delta R_{x x}$, of the InSe-capped section of the graphene Hall bar after illumination. 


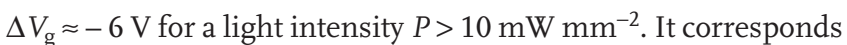
to an increase in the electron density by $\Delta n=4.5 \times 10^{11} \mathrm{~cm}^{-2}$ at $V_{\mathrm{g}}=0 \mathrm{~V}$ (Supporting Information S1).

The photosensivity of the InSe-capped graphene section of the device is illustrated in more detail in Figure 1d, which shows the $V_{\mathrm{g}}$-dependence of the light-induced change of $R_{x x}$, i.e., $\Delta R_{x x}=R_{x x}($ light $)-R_{x x}$ (dark), at $T=2 \mathrm{~K} . \Delta R_{x x}$ has a maximum at a gate voltage $V_{\mathrm{L}} \approx 0 \mathrm{~V}$, passes through zero at a gate voltage $V_{F} \approx+6 \mathrm{~V}$ and changes sign with a minimum at $V_{\mathrm{D}} \approx+10 \mathrm{~V}$. The values of $V_{\mathrm{D}}$ and $V_{\mathrm{L}}$ correspond approximately to the peak positions of the $R_{x x}\left(V_{\mathrm{g}}\right)$ curves in darkness and under illumination, respectively (Figure 1c). At the peak of $R_{x x}\left(V_{\mathrm{g}}\right)$ the Fermi energy, $E_{\mathrm{F}}$, aligns with the neutrality point of the graphene's band structure in darkness $\left(V_{\mathrm{D}} \approx+10 \mathrm{~V}\right)$ and under illumination $\left(V_{\mathrm{L}} \approx 0 \mathrm{~V}\right)$. The dependence of $R_{x x}$ and $\Delta R_{x x}$ on $V_{\mathrm{g}}$ is weakly affected by temperature, revealing similar features at both $T=2$ and $300 \mathrm{~K}$ (Figure 1c and Supporting Information S1).

The data in Figure 1 show that in darkness the capping of graphene with InSe increases significantly the electron density in the graphene layer. Under illumination, the density of electrons in the InSe-capped graphene increases further. In particular, the similar form of the $R_{x x}\left(V_{\mathrm{g}}\right)$ curves at low and room temperature indicates a relatively weak contribution of phonon scattering to the mobility of carriers. This behavior can be accounted for by the dominant contribution of carrier scattering by negatively charged acceptor impurities in our chemical vapor deposition (CVD)-graphene. ${ }^{[13]}$ In the following sections, we use gate-dependent quantum Hall effect measurements to examine how the photogenerated electrons are distributed between the component layers of the InSe/graphene heterostructure.

\subsection{Landau Level Quantization and Photoquantum Hall Effect}

The effects of light on the Hall resistance, $R_{x y}$, and its dependence on $V_{\mathrm{g}}$ are shown in Figure 2a for various magnetic fields, $B$, and $T=2 \mathrm{~K}$. For gate voltages in the range $V_{\mathrm{L}}<V_{\mathrm{g}}<V_{\mathrm{D}}$ (see shaded areas), the optical excitation induces a sign reversal of the Hall voltage and of the QH plateau, $R_{x y}=h / v e^{2}$, where $v=2$ is the graphene Landau level (LL) filling factor. Thus, illumination induces a transition from a hole- to an electroncarrier current in the graphene layer over an extended range of gate voltages (Figure $2 \mathrm{~b}$ ).

We probe the distribution of charge in the graphene and InSe layers in the dark and under illumination by analyzing the magnetoresistance $R_{x x}\left(V_{\mathrm{g}}\right)$ curves of the InSe-capped graphene layer, see Figure 3a,b. At low temperature $(T=2 \mathrm{~K})$ and in the presence of a quantizing magnetic field, both in darkness (left panel, Figure 3a) and under illumination (right panel, Figure $3 \mathrm{a})$, the plots of $R_{x x}\left(V_{\mathrm{g}}\right)$ display a pair of minima over a range of gate voltages corresponding to a quantized value of $R_{x y}=h / 2 e^{2}$. Furthermore, under illumination, the minima in $R_{x x}$ and the corresponding width of the QH plateaux in $R_{x y}$ become asymmetric, and the $\mathrm{QH}$ plateau at $V_{\mathrm{g}}>0 \mathrm{~V}$ becomes almost twice as wide as in darkness (Figure $3 b$ ).

For single layer graphene, the energy-level spectrum of Dirac fermions in a magnetic field is described by the relation

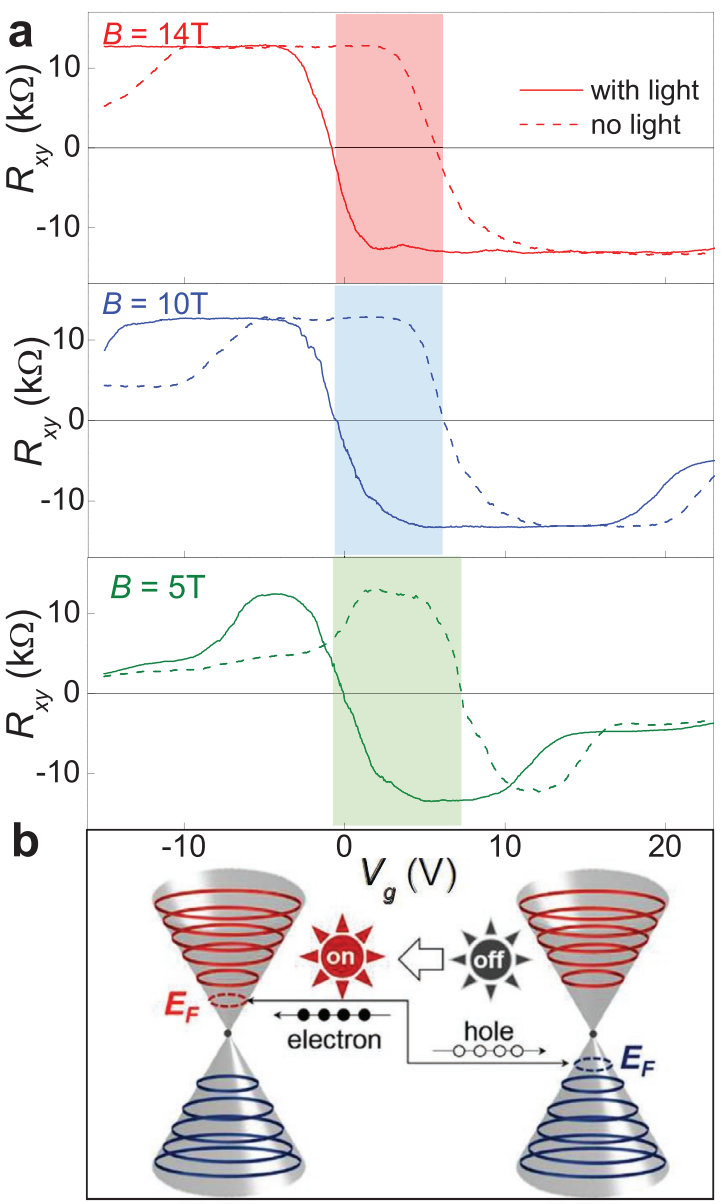

Figure 2. a) Hall resistance, $R_{x y}$, of InSe-capped graphene versus gate voltage, $V_{\mathrm{g}}$, in darkness (dashed line) and under illumination (continuous line) at $T=2 \mathrm{~K}$ and $B=5,10$, and $14 \mathrm{~T}(I=0.5 \mu \mathrm{A}, \lambda=633 \mathrm{~nm}$, and $P=9 \mathrm{~mW} \mathrm{~mm} \mathrm{~m}^{-2}$ ). The shaded areas identify a range of gate voltages for which light reverses the sign of $R_{x y}$. b) Landau level quantization in graphene and sketch of the movement of the Fermi level from the lower to the upper part of the Dirac cone under illumination, leading to the sign reversal of $R_{x y}$ shown in part (a).

$E_{i}=\operatorname{sgn}(i) \sqrt{e h v_{\mathrm{F}}^{2} B|i| / \pi}$, where $v_{\mathrm{F}} \approx 10^{6} \mathrm{~m} \mathrm{~s}^{-1}$ is the Fermi velocity and $i=0, \pm 1, \pm 2 \ldots$ The spectrum comprises electron $(i \geq 1)$ and hole $(i \leq-1)$ LLs, as well as a LL $(i=0)$ at the neutrality point (inset of Figure 3a). ${ }^{[20,21]}$ Since the LLs for electrons and holes are symmetrically arranged around the $i=0$ state, one might expect an approximately symmetric $V_{\mathrm{g}}$-dependence of $R_{x x}$ and $R_{x y}$ relative to the voltage position of the peak in $R_{x x}\left(V_{\mathrm{g}}\right)$. For graphene or InSe-capped graphene in darkness, the minima in $R_{x x}$ and the corresponding width of the $\mathrm{QH}$ plateaux in $R_{x y}$ at the LL filling factor $v=2$ are indeed symmetric. However, for InSecapped graphene under illumination, both peaks and plateaux become asymmetric (Figure 3b).

For the case of uncapped graphene and InSe-capped graphene in darkness, we use a simple capacitance equation, i.e., $C=e\left[\mathrm{~d} n_{\mathrm{G}} / \mathrm{d} V_{\mathrm{g}}\right]$, to calculate the voltage separation $\Delta V_{\mathrm{g}}$ of the maxima in the $R_{x x}\left(V_{\mathrm{g}}\right)$ curve at different $B$. Here $C=\varepsilon \varepsilon_{0} / t$ is the "classical" capacitance per unit area of the graphene/ $/ \mathrm{SiO}_{2} /$ Si heterostructure, $e$ is the elementary charge, $t=285 \mathrm{~nm}$ is the 

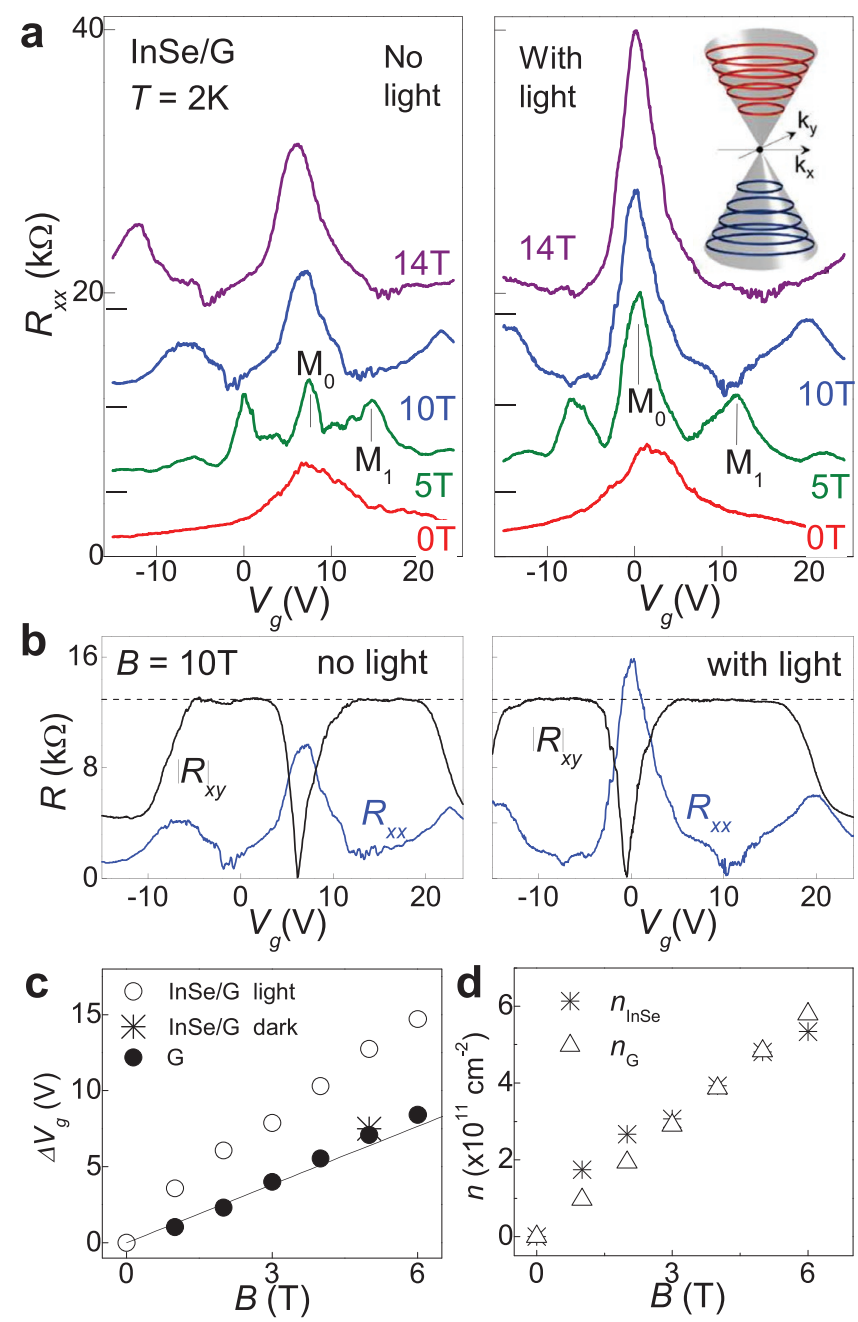

Figure 3. a) Resistance, $R_{x x}$, of InSe-capped graphene versus gate voltage, $V_{g}$, at various applied magnetic fields $B$ in darkness (left) and under illumination (right) $\left(T=2 \mathrm{~K}, \lambda=633 \mathrm{~nm}\right.$, and $\left.P=9 \mathrm{~mW} \mathrm{~mm}{ }^{-2}\right)$. For clarity, curves at different $B$ are displaced along the vertical axis. $M_{0}$ and $M_{1}$ label two maxima in $R_{x x}\left(V_{g}\right)$ corresponding to the alignment of the Fermi level with the $i=0$ and $i=+1$ Landau levels (LLs). Inset: LL quantization in graphene. b) $R_{x x}$ and modulus of the Hall resistance, $\left|R_{x y}\right|$, versus $V_{\mathrm{g}}$ in darkness (left) and under illumination (right) at $B=10 \mathrm{~T}$. Dashed lines show $R_{x y}=h / 2 e^{2}$. c) B-dependence of the separation, $\Delta V_{g}$, between peaks in $R_{x x}\left(V_{\mathrm{g}}\right), \mathrm{M}_{0}$ and $\mathrm{M}_{1}$, for graphene $(\mathrm{G})$ and InSe-capped graphene $(\mathrm{InSe} / \mathrm{G})$ in darkness and under illumination $(T=2 \mathrm{~K})$. The line is the calculated dependence for graphene according to the model described in the text. d) Density of carriers in graphene $\left(n_{\mathrm{C}}\right)$ and donor states in InSe $\left(n_{\text {InSe }}\right)$ versus $B$ for InSe-capped graphene.

$\mathrm{SiO}_{2}$ layer thickness, $\varepsilon=3.9$ is the relative dielectric constant of $\mathrm{SiO}_{2}, \varepsilon_{0}$ is the permittivity of free space, and $n_{\mathrm{G}}$ is the carrier density in the graphene layer. In our model, we have neglected the quantum capacitance, $C_{\mathrm{q}}$, of graphene, which accounts for the nonclassical dependence of $n_{\mathrm{G}}$ on the applied gate voltage when the Fermi energy, $E_{\mathrm{F}}$, is close to the low density of states at the Dirac point: $C_{\mathrm{q}}=2 e^{2} E_{\mathrm{F}} / \pi \hbar^{2} v_{\mathrm{F}}^{2}$. When the Fermi level is far from the Dirac point, the effect of the quantum capacitance becomes negligible (Supporting Information S4).

Over the range of $V_{\mathrm{g}}$ between the maxima $\mathrm{M}_{0}$ and $\mathrm{M}_{1}$ in $R_{x x}\left(V_{\mathrm{g}}\right)$ (see Figure 3a), corresponding respectively to the alignment of

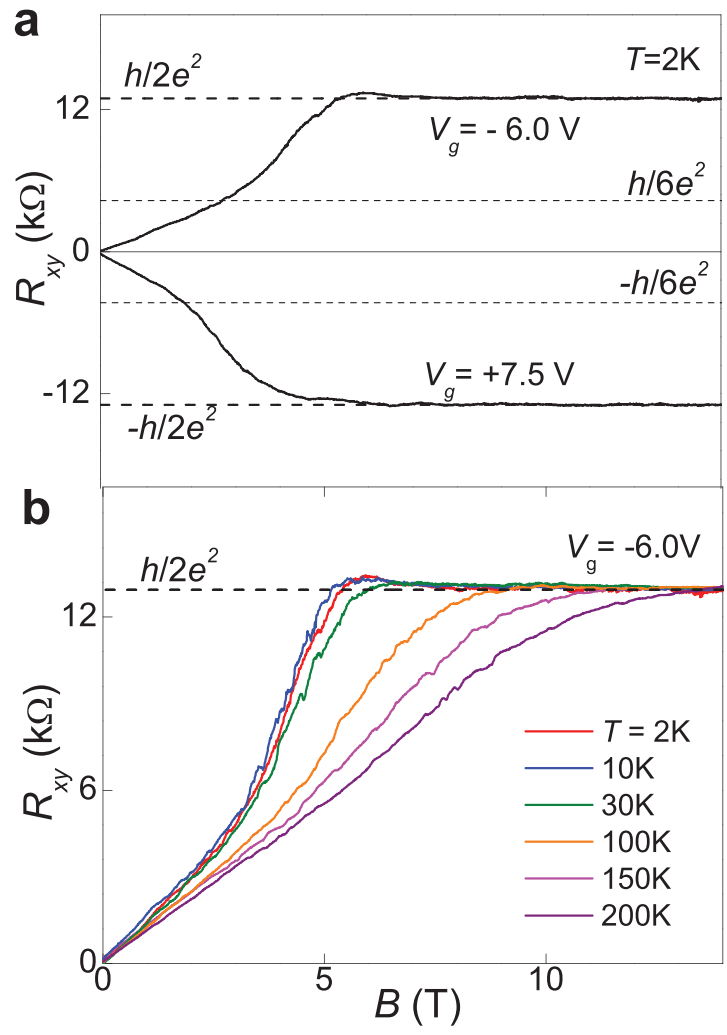

Figure 4. a) Hall resistance, $R_{x y}$, of InSe-capped graphene versus magnetic field $B$ at $T=2 \mathrm{~K}$ and different gate voltages $V_{\mathrm{g}}$ under illumination $\left(I=0.5 \mu \mathrm{A}, \lambda=633 \mathrm{~nm}\right.$, and $\left.P=9 \mathrm{~mW} \mathrm{~mm}^{-2}\right)$. b) Hall resistance $R_{x y}$ of InSe-capped graphene versus $B$ under illumination at different temperatures, $T$, and $V_{\mathrm{g}}=-6.0 \mathrm{~V}\left(I=0.5 \mu \mathrm{A}, \lambda=633 \mathrm{~nm}\right.$, and $\left.P=9 \mathrm{~mW} \mathrm{~mm} \mathrm{~mm}^{-2}\right)$.

the Fermi level with the $i=0$ and $i=+1 \mathrm{LLs}, \Delta V_{\mathrm{g}}=\mathrm{eg} / C$, where $g=4 e B / h$ is the LL degeneracy. ${ }^{[20]}$ This simple model reproduces quite accurately the data for the uncapped graphene in darkness and under illumination at different values of $B$ and also for InSe-capped graphene in darkness (Figure 3c, black dots and star). However, it does not reproduce the values of $\Delta V_{\mathrm{g}}$ in InSecapped graphene under illumination (Figure $3 c$, open circles). To explain this behavior, we note that when the positive gate voltage or the intensity of light are sufficiently large that the Fermi level approaches the InSe CB edge, electrons start to fill donor states below the $\mathrm{CB}$ edge. In this case the carrier density induced by $V_{\mathrm{g}}$ has two contributions, one from the InSe layer $\left(n_{\text {Inse }}\right)$ and one from graphene $\left(n_{\mathrm{G}}\right)$. From the measured value of $\Delta V_{\mathrm{g}}$, corresponding to the separation of the peaks $\mathrm{M}_{0}$ and $\mathrm{M}_{1}$ peaks in $R_{x x}$, we estimate $n_{\mathrm{InSe}}$, i.e., $e n_{\mathrm{InSe}}=C \Delta V_{g}-e n_{\mathrm{G}}$, where $n_{\mathrm{G}}=4 e B / h$ and $C=\varepsilon \varepsilon_{0} / t$. The values of $n_{\text {InSe }}$ are plotted in Figure $4 \mathrm{~d}$ for different $B: n_{\text {Inse }}$ increases with $B$ from zero at $B=0 \mathrm{~T}$ and $V_{\mathrm{g}} \approx 0 \mathrm{~V}$ to $6 \times 10^{11} \mathrm{~cm}^{-2}$ at $B=6 \mathrm{~T}$ and $V_{\mathrm{g}} \approx 15 \mathrm{~V}$. These values are comparable to the electron density in the $i=+1 \mathrm{LL}$ of the graphene layer, $n_{\mathrm{G}}=4 e B / h$, also shown in Figure $3 \mathrm{~d}$. The increase of $n_{\text {InSe }}$ with $B$ corresponds to an increase of the Fermi energy from the Dirac point at $E_{0}=0$ to the $i=+1 \mathrm{LL}$ at $E_{1}=\sqrt{\operatorname{eh} v_{\mathrm{F}}^{2} B / \pi}$. Thus, at low temperatures, a significant fraction of electrons become bound on the relatively large density of donors $\left(\approx 10^{11} \mathrm{~cm}^{-2}\right)$ in the InSe capping layer. These donor states are likely to extend over a broad energy range: we estimate that $E_{1}-E_{0} \approx 60 \mathrm{meV}$ at 
$B=10 \mathrm{~T}$. The binding energy of isolated donors in bulk InSe is $E_{\mathrm{d}} \approx 30 \mathrm{meV} .^{[17]}$ However, for thin InSe, $E_{\mathrm{d}}$ depends on the position of the donors relative to the surface; furthermore, closely spaced donors can have larger binding energies. At low temperatures $(T<100 \mathrm{~K})$ the electrons in InSe remain bound to donors so that the QH voltage of the graphene layer is not "short-circuited" by the parallel conduction in the InSe layer.

The larger negative charge in the InSe-capped graphene with increasing positive gate voltage and/or increasing light intensity causes an asymmetric distribution of LL-features and $\mathrm{QH}$ plateaux relative to the peak in $R_{x x}\left(V_{\mathrm{g}}\right)$ (see Figure $3 \mathrm{~b}$ ). In particular, over a range of gate biases the $\mathrm{QH}$ plateau extends to high $B$. For example, at $V_{\mathrm{g}}=7.5 \mathrm{~V}$ and $V_{\mathrm{g}}=-6.0 \mathrm{~V}$, the $\mathrm{QH}$ plateau extends from $B \approx 5$ to $14 \mathrm{~T}$ at $T=2 \mathrm{~K}$, corresponding to an increase in sheet density of $\approx 5 \times 10^{11} \mathrm{~cm}^{-2}$ (Figure $4 \mathrm{a}$ ), and persists over a wide range of temperatures (Figure $4 \mathrm{~b}$ ). We have observed this behavior for several devices although the $\mathrm{QH}$ resistance plateau occurs for gate voltages that vary slightly from sample to sample (Supporting Information S2). The extended $v=2 \mathrm{QH}$ plateau arises from the "pinning" of the chemical potential in the energy gap between Landau levels when their degeneracy increases with increasing $B$. This is facilitated by the close alignment of the conduction band edge of InSe with the Dirac point of graphene: the electron affinity of graphene $\left(\chi_{\mathrm{Gr}}=4.5 \mathrm{eV}\right)$ is only slightly smaller than that of bulk $\operatorname{InSe}\left(\chi_{\text {InSe }}=4.6 \mathrm{eV}\right) .{ }^{[13]}$

The extended $\mathrm{QH}$ plateau reported here is similar in form to that for graphene grown epitaxially by the thermal decomposition of the surface layer of SiC.[22-26] This phenomenon has been explained in terms of the transfer of charge carriers, between the graphene layer and defects in the adjacent SiC substrate. ${ }^{[26]}$ The data reported here demonstrate that a similar process occurs between graphene and donors in the n-type InSe capping layer. Thus the $i=0$ Landau level at the Dirac point of the graphene layer remains completely filled when its degeneracy increases with increasing $B$. Our data illustrate that this effect can be controlled by both the gate voltage and optical illumination. Although the $v=2$ plateau in $R_{x y}$ becomes weaker with increasing temperature, it can be seen even up to temperatures of $T \approx 200 \mathrm{~K}$ at $B=14 \mathrm{~T}$ (Figure $4 \mathrm{~b}$ ). We attribute the quenching of the $\mathrm{QH}$ plateau at higher $T$ to the thermal ionization of electrons out of the donor states into the conduction band of the InSe capping layer, leading to thermally activated parallel conduction in InSe (Supporting Information S3).

\subsection{Photocurrent Maps of InSe-Graphene Heterostructures}

To investigate in further detail the light-induced charge transfer at the InSe/graphene interface, we carried out a series of measurements to produce spatially resolved photocurrent maps of graphene-InSe-graphene planar devices. As shown in Figure 5a, in these devices the two graphene $(\mathrm{G})$ layers serve as source (s) and drain (d) electrodes to an InSe channel of length $l \approx 1 \mu \mathrm{m}$ and width $w \approx 20 \mu \mathrm{m}$. The InSe layer is encapsulated with a hBN layer, which also overlaps the two graphene layers. The room temperature photocurrent map is obtained by scanning a focused laser beam across the plane of the device with or without an applied source-drain bias voltage, $V_{\text {sd }}$. As shown in Figure 5b, for $V_{\text {sd }}=0$, a photocurrent $\Delta I$ is generated in the InSe-capped graphene layers (see areas within the dashed lines in Figure $5 \mathrm{~b}$ ). This photocurrent is nonuniform, is enhanced at the graphene edges and has opposing polarities on the two graphene electrodes. These data indicate the presence of a built-in electric field at the graphene/InSe interface (bottom inset, Figure $5 \mathrm{~b}$ ) and that this field is stronger at the edges of the graphene electrodes. As shown in Figure $5 c$, the photocurrent increases for $V_{\mathrm{sd}}>0$ : the photocurrent is generated in two distinct regions: the area of the InSe flake between the two graphene electrodes where the in-plane electric field $F \neq 0$ (region $A$ ) and in the InSe-capped graphene layers where $F=0$ (region B). No significant photocurrent is observed in the graphene regions that are not capped by the InSe layer. We ascribe the large photocurrent in region $\mathrm{A}$ to the drift of photogenerated carriers under the action of the applied electric field in the InSe gap between the graphene electrodes (bottom inset, Figure 5c). We assign the smaller, but significant photocurrent in the InSe-capped graphene layers (region $B$ in Figure $5 c$ ) to thermal diffusion: the photogenerated carriers diffuse in InSe and are extracted at the graphene electrodes by charge transfer. These data demonstrate that a large photoresponse can be achieved in regions $\mathrm{B}$ with distinct light-absorbing (InSe) and conducting (graphene) layers, and regions $\mathrm{A}$ where the InSe layer acts simultaneously as a light-absorbing and conducting medium. The charge-transfer mechanism for photodetection in regions B is somewhat different from that previously reported for InSe-graphene planar and vertical photodetector structures ${ }^{[27]}$ and analogous to that demonstrated in pentacene-graphene heterostructures. ${ }^{[28]}$ The light-induced transfer of charge between InSe and graphene offers an effective method to increase or decrease the carrier density in graphene, causing a change in its resistance and quantum Hall effect that are gate-controllable and only weakly dependent on temperature.

From the measured values of $\Delta I$ for different incident powers, $P$, with an unfocused laser beam (i.e., illuminating the whole area of the device), we estimate a photoresponsivity $R=\Delta I / P$ of up to $R \approx 10^{6} \mathrm{~A} \mathrm{~W}^{-1}$ at the lowest incident power $P \approx 10^{-5} \mathrm{~W} \mathrm{~m}^{-2}(\lambda=633 \mathrm{~nm})$ with $V_{\mathrm{sd}}=0.1 \mathrm{~V}$. The photocurrent map in Figure $5 \mathrm{c}$ shows that both regions $\mathrm{A}$ and $\mathrm{B}$ contribute to the photoresponse of the device. Also, the photoresponse is relatively fast with rise and decay times of the photocurrent of less than $1 \mathrm{~ms}$ in both regions A and B (Figure 5d). By comparing the photocurrent signals in areas with and without the hBN layer, we find that the encapsulation of InSe by hBN induces a systematic increase of the signal (approximately by a factor of 3). We attribute this behavior, which is not observed when the hBN layer lies below the InSe layer, to an increased absorption of light by the InSe layer due to the reduced reflection of the laser light at the interface between air and hBN (refractive index $\left.n_{\mathrm{hBN}}=1.8\right),{ }^{[29]}$ compared to air $\left(n_{\text {air }}=1\right)$ and InSe $\left(n_{\text {InSe }}=2.7\right) \cdot{ }^{[30]}$ The encapsulation of InSe by hBN also leads to an increased photoluminescence and Raman signals of InSe. In summary, these photocurrent mapping experiments demonstrate that light-induced charge-transfer at the InSe/graphene interface acts to photosensitize the graphene layer, thus enhancing its photoresponse. The response time of the photocurrent is relatively slow and is partly limited by charge traps 
a
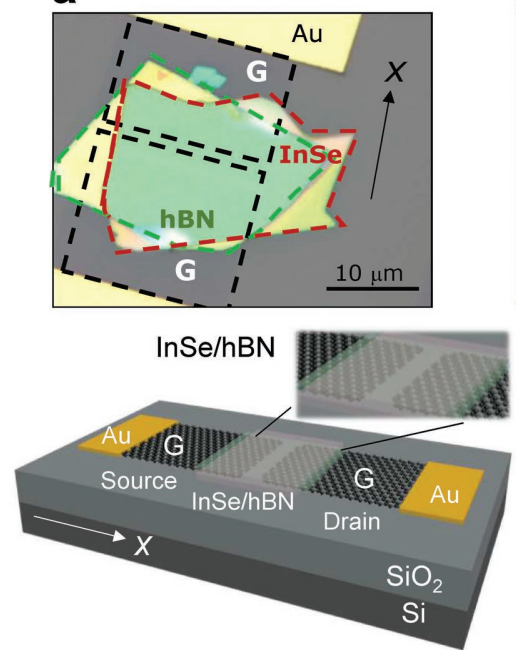

b
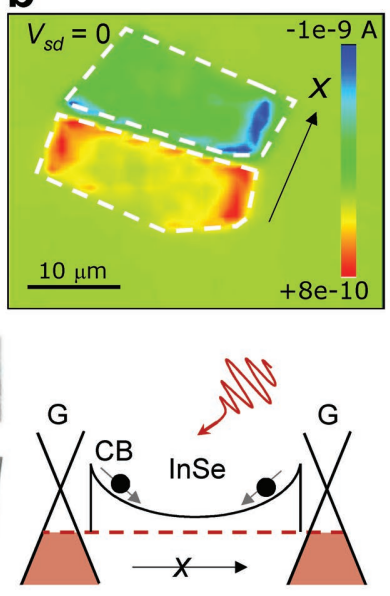

C
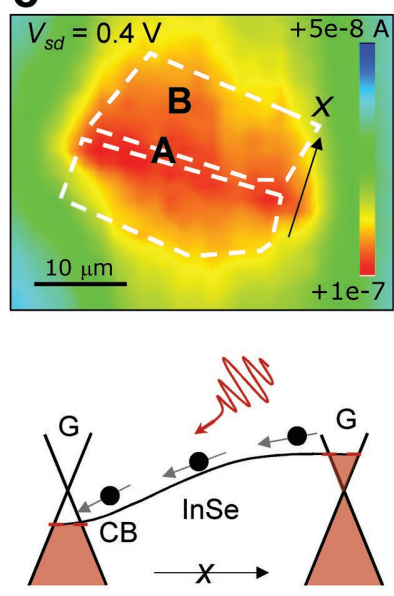
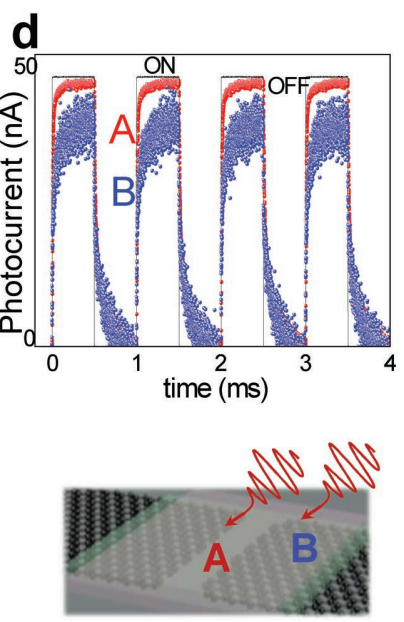

Figure 5. a) Optical image and diagram of a graphene-InSe/hBN-graphene planar device with regions of overlaps between the InSe (red line), hBN (green lines) and graphene (black lines) layers. Photocurrent maps acquired b) without and c) with an applied source-drain voltage, $V_{\text {sd }}$, between two graphene electrodes at $T=300 \mathrm{~K}(\lambda=405 \mathrm{~nm}$ and $P=50 \mu \mathrm{W})$. In part (b), the photocurrent $\Delta /$ is generated in the region of overlap between the InSe and graphene electrodes (indicated by dashed white lines) and is enhanced at the graphene edges $\left(V_{\mathrm{sd}}=0.4 \mathrm{~V}\right.$ ). In part (c), the photocurrent $\Delta /$ is generated in the gap between the graphene electrodes (region A) and in the InSe-capped graphene layers (region B). Insets: photocurrent generation b) by the built-in electric field at the graphene/InSe interface and c) by drift (or thermal diffusion) of photogenerated carriers. $d$ ) Temporal dependence of the photocurrent in regions $A$ and $B$.

and the $R C$ time of our device structures. All measurements presented in this work are obtained under quasistatic conditions so are not influenced by dynamic polarization effects. To probe the role of dipole-dipole interactions on the photoexcited charge transfer, ultrafast pump-probe optical spectroscopy studies and optimized device geometries are required.

\section{Conclusions}

In conclusion, we have demonstrated novel hybrid InSe/graphene phototransistor devices. By mounting the InSe/graphene heterostructure on an oxidized silicon substrate, we have produced devices in which the electronic properties of the graphene layer can be controlled by a combination of field effect gating and the transfer of photoexcited carriers across the InSe-graphene interface. In this way, the Fermi energy of the graphene layer can be fine-tuned across the Dirac point, thus achieving either $\mathrm{n}$ - or $\mathrm{p}$-type conduction. The light-induced charge transfer also induces a reversal of the sign of the Hall voltage and of the $h / 2 e^{2}$ quantum Hall plateau, which extends over a wide range of applied magnetic fields and temperatures. We have measured the carrier density in the InSe and graphene layers under optical excitation. Our results demonstrate the important role of the donors in the InSe layer in determining the optical and quantum Hall characteristics of this type of device. Our hybrid phototransistor combine the high photosensitivity of InSe with the unique electrical properties of graphene. These devices are made by a relatively simple fabrication process and offer a flexible route to integration with existing Si-manufacturing technologies. Prospects for further research include the investigation of the charge transfer in new multilayered vdW heterostructures, for example in InSe/hBN/graphene for controlled charge transfer between graphene and InSe through an intermediate tunnel hBN barrier. In particular, the broadband absorption of InSe including a range of intraband and interband optical transitions in the visible, near-infrared and infrared range ${ }^{[7,9]}$ provide an opportunity to tune the photoresponsivity of graphene and unlock its potential for optoelectronic applications, beyond the capability of traditional semiconductor devices and other $2 \mathrm{D}$ photodetectors in the current literature. ${ }^{[1,31-33]}$

\section{Experimental methods}

The single layer graphene was grown by CVD on a copper foil substrate and was transferred to a $\mathrm{SiO}_{2} / p$-Si substrate $\left(\mathrm{SiO}_{2}\right.$ layer thickness $t=285 \mathrm{~nm})$. The graphene was shaped into a multiterminal Hall bar using electron-beam lithography and oxygen/argon reactive ion etching. Au/Ti electrodes $(100 / 10 \mathrm{~nm})$ were then evaporated on the graphene layer. High quality InSe flakes were exfoliated from a Bridgman-grown InSe crystal onto a polydimethylsiloxane membrane. A selected InSe flake was dry transferred to the graphene Hall bar, capping a section of the graphene channel, but not contacting the $\mathrm{Au} / \mathrm{Ti}$ electrodes. Exfoliated InSe flakes capped with hBN were also integrated into two-terminal planar device structures incorporating graphene electrodes.

The magneto-transport measurements were performed with the device mounted inside a superconducting magneto-cryostat at magnetic fields $B$ up to $14 \mathrm{~T}$ or using a resistive magnet with $B$ up to $25 \mathrm{~T}$ (Supporting Information S2) and over a range of gate voltages, $V_{\mathrm{g}}$, applied between the graphene and the Si-substrate. For the magnetotransport experiments under optical illumination, an unfocused $\mathrm{He}-\mathrm{Ne}$ laser beam (wavelength $\lambda=632.8 \mathrm{~nm}$, power, $P$, up to $13 \mathrm{~mW} \mathrm{~mm}$, and spot diameter $d \approx 1 \mathrm{~mm}$ ) was used. For the photoconductivity mapping experiments, a focused laser beam (wavelength $\lambda=405 \mathrm{~nm}$ and spot diameter $d \approx 1 \mu \mathrm{m}$ ) was used. 


\section{Supporting Information}

Supporting Information is available online from the Wiley Online Library or from the author.

\section{Acknowledgements}

This work was supported by the Engineering and Physical Sciences Research Council [grant number EP/M012700/1]; the European Magnetic Field Laboratory (EMFL); the Engineering and Physical Sciences Research Council via its membership to the EMFL [grant number EP/N01085X/1]; the European Research Council (ERC) under the European Union's Horizon 2020 research and innovation program [grant agreement number 696656]; The University of Nottingham; The National Academy of Sciences of Ukraine; The Leverhulme Trust [RF-2017-224]; The Royal Society [IE160395]; and the Chinese Academy of Sciences. E.E.V. acknowledges support from the Russian Science Foundation (17-12-01393).

\section{Conflict of Interest}

The authors declare no conflict of interest.

\section{Keywords}

graphene, InSe, quantum Hall effect

Received: August 8, 2018

Revised: October 31, 2018

Published online: November 27, 2018

[1] A. C. Ferrari, F. Bonaccorso, V. I. Fal'ko, K. S. Novoselov, S. Roche, P. Bøggild, S. Borini, F. H. L. Koppens, V. Palermo, N. Pugno, J. A. Garrido, R. Sordan, A. Bianco, L. Ballerini, M. Prato, E. Lidorikis, J. Kivioja, C. Marinelli, T. Ryhänen, A. Morpurgo, J. N. Coleman, V. Nicolosi, L. Colombo, A. Fert, M. Garcia-Hernandez, A. Bachtold, G. F. Schneider, F. Guinea, C. Dekker, M. Barbone, Z. Sun, C. Galiotis, A. N. Grigorenko, G. Konstantatos, A. Kis, M. Katsnelson, L. Vandersypen, A. Loiseau, V. Morandi, D. Neumaier, E. Treossi, V. Pellegrini, M. Polini, A. Tredicucci, G. M. Williams, B. H. Hong, J.-H. Ahn, J. M. Kim, H. Zirath, B. J. van Wees, H. van der Zant, L. Occhipinti, A. D. Matteo, I. A. Kinloch, T. Seyller, E. Quesnel, X. Feng, K. Teo, N. Rupesinghe, P. Hakonen, S. R. T. Neil, Q. Tannock, T. Löfwander, J. Kinaret, Nanoscale 2015, 7, 4598.

[2] A. K. Geim, I. V. Grigorieva, Nature 2013, 499, 419.

[3] L. Ci, L. Song, C. Jin, D. Jariwala, D. Wu, Y. Li, A. Srivastava, Z. F. Wang, K. Storr, L. Balicas, F. Liu, P. M. Ajayan, Nat. Mater. 2010, 9, 430.

[4] J. Yang, S. Tran, J. Wu, S. Che, P. Stepanov, T. Taniguchi, K. Watanabe, H. Baek, D. Smirnov, R. Chen, C. N. Lau, Nano Lett. 2018, 18, 229.

[5] Q. H. Wang, K. Kalantar-Zadeh, A. Kis, J. N. Coleman, M. S. Strano, Nat. Nanotechnol. 2012, 7, 699.

[6] K. Wang, K. De Greve, L. A. Jauregui, A. Sushko, A. High, Y. Zhou, G. Scuri, T. Taniguchi, K. Watanabe, M. D. Lukin, H. Park, P. Kim, Nat. Nanotechnol. 2018, 13, 128.

[7] G. W. Mudd, M. R. Molas, X. Chen, V. Zólyomi, K. Nogajewski, Z. R. Kudrynskyi, Z. D. Kovalyuk, G. Yusa, O. Makarovsky, L. Eaves, M. Potemski, V. I. Fal'ko, A. Patanè, Sci. Rep. 2016, 6, 39619.

[8] M. Brotons-Gisbert, D. Andres-Penares, J. Suh, F. Hidalgo, R. Abargues, P. J. Rodríguez-Cantó, A. Segura, A. Cros, G. Tobias, E. Canadell, P. Ordejón, J. Wu, J. P. Martínez-Pastor, J. F. Sánchez-Royo, Nano Lett. 2016, 16, 3221.

[9] D. A. Bandurin, A. V. Tyurnina, G. L. Yu, A. Mishchenko, V. Zólyomi, S. V. Morozov, R. K. Kumar, R. V. Gorbachev, Z. R. Kudrynskyi,
S. Pezzini, Z. D. Kovalyuk, U. Zeitler, K. S. Novoselov, A. Patanè, L. Eaves, I. V. Grigorieva, V. I. Fal'ko, A. K. Geim, Y. Cao, Nat. Nanotechnol. 2017, 12, 223.

[10] W. Feng, W. Zheng, W. Cao, P. A. Hu, Adv. Mater. 2014, 26, 6587.

[11] M. Hamer, E. Tóvári, M. Zhu, M. D. Thompson, A. Mayorov, J. Prance, Y. Lee, R. P. Haley, Z. R. Kudrynskyi, A. Patanè, D. Terry, Z. D. Kovalyuk, K. Ensslin, A. V. Kretinin, A. Geim, R. Gorbachev, Nano Lett. 2018, 18, 3950.

[12] N. Balakrishnan, Z. R. Kudrynskyi, E. F. Smith, M. W. Fay, O. Makarovsky, Z. D. Kovalyuk, L. Eaves, P. H. Beton, A. Patanè, 2D Mater. 2017, 4, 025043

[13] Z. R. Kudrynskyi, M. A. Bhuiyan, O. Makarovsky, J. D. G. Greener, E. E. Vdovin, Z. D. Kovalyuk, Y. Cao, A. Mishchenko, K. S. Novoselov, P. H. Beton, L. Eaves, A. Patanè, Phys. Rev. Lett. 2017, 119, 157701.

[14] Z. Chen, J. Biscaras, A. Shukla, Nanoscale 2015, 7, 5981.

[15] A. A. Kistanov, Y. Cai, K. Zhou, S. V. Dmitriev, Y.-W. Zhang, Phys. Chem. Chem. Phys. 2018, 20, 12939.

[16] S. Zhou, C.-C. Liu, J. Zhao, Y. Yao, npj Quantum Mater. 2018, https://doi.org/10.1038/s41535-018-0089-0.

[17] G. W. Mudd, A. Patanè, Z. R. Kudrynskyi, M. W. Fay, O. Makarovsky, L. Eaves, Z. D. Kovalyuk, V. Zólyomi, V. I. Fal'ko, Appl. Phys. Lett. 2014, 105, 221909.

[18] Y. Lee, R. Pisoni, H. Overweg, M. Eich, P. Rickhaus, A. Patanè, Z. R. Kudrynskyi, Z. D. Kovalyuk, R. Gorbachev, K. Watanabe, T. Taniguchi, T. Ihn, K. Ensslin, 2D Mater. 2018, 5, 035040.

[19] Y. Sun, S. Luo, X.-G. Zhao, K. Biswas, S.-L. Li, L. Zhang, Nanoscale 2018, 10, 7991

[20] K. S. Novoselov, A. K. Geim, S. V. Morozov, D. Jiang, M. I. Katsnelson, I. V. Grigorieva, S. V. Dubonos, A. A. Firsov, Nature 2005, 438, 197.

[21] Y. Zhang, Y.-W. Tan, H. L. Stormer, P. Kim, Nature 2005, 438, 201.

[22] A. Tzalenchuk, S. Lara-Avila, A. Kalaboukhov, S. Paolillo, M. Syväjärvi, R. Yakimova, O. Kazakova, T. J. B. M. Janssen, V. I. Fal'ko, S. Kubatkin, Nat. Nanotechnol. 2010, 5, 186.

[23] S. Kopylov, A. Tzalenchuk, S. Kubatkin, V. I. Fal'ko, Appl. Phys. Lett. 2010, 97, 112109 .

[24] T. J. B. M. Janssen, A. Tzalenchuk, S. Lara-Avila, S. Kubatkin, V. I. Fal'ko, Rep. Prog. Phys. 2013, 76, 104501.

[25] R. Ribeiro-Palau, F. Lafont, J. Brun-Picard, D. Kazazis, A. Michon, F. Cheynis, O. Couturaud, C. Consejo, B. Jouault, W. Poirier, F. Schopfer, Nat. Nanotechnol. 2015, 10, 965.

[26] J. A. Alexander-Webber, J. Huang, D. K. Maude, T. J. B. M. Janssen, A. Tzalenchuk, V. Antonov, T. Yager, S. Lara-Avila, S. Kubatkin, R. Yakimova, R. J. Nicholas, Sci. Rep. 2016, 6, 30296.

[27] G. W. Mudd, S. A. Svatek, L. Hague, O. Makarovsky, Z. R. Kudrynskyi, C. J. Mellor, P. H. Beton, L. Eaves, K. S. Novoselov, Z. D. Kovalyuk, E. E. Vdovin, A. J. Marsden, N. R. Wilson, A. Patanè, Adv. Mater. 2015, 27, 3760

[28] X. Chen, X. Liu, B. Wu, H. Nan, H. Guo, Z. Ni, F. Wang, X. Wang, Y. Shi, X. Wang, Nano Lett. 2017, 17, 6391.

[29] S. L. Rumyantsev, M. E. Levinshtein, A. D. Jackson, S. N. Mohammad, G. L. Harris, M. G. Spencer, M. S. Shur, in Properties of Advanced Semiconductor Materials GaN, AIN $\ln N, B N$, SiC, SiGe (Eds: M. E. Levinshtein, S. L. Rumyantsev, M. S. Shur), John Wiley \& Sons, Inc., New York 2001, pp. 67-92.

[30] F. J. Manjón, Y. van der Vijver, A. Segura, V. Muñoz, Semicond. Sci. Technol. 2000, 15, 806.

[31] F. H. L. Koppens, T. Mueller, Ph. Avouris, A. C. Ferrari, M. S. Vitiello, M. Polini, Nat. Nanotechnol. 2014, 9, 780 .

[32] F. Yan, L. Zhao, A. Patanè, P. A. Hu, X. Wei, W. Luo, D. Zhang, Q. Lv, Q. Feng, C. Shen, K. Chang, L. Eaves, K. Wang, Nanotechnology 2017, 28, 27LTO1.

[33] S. Goossens, G. Navickaite, C. Monasterio, S. Gupta, J. J. Piqueras, R. Pérez, G. Burwell, I. Nikitskiy, T. Lasanta, T. Galán, E. Puma, A. Centeno, A. Pesquera, A. Zurutuza, G. Konstantatos, F. Koppens, Nat. Photonics 2017, 11, 366. 